\title{
L'agriculture durable en système de grandes cultures : l'exemple des plateaux du sud-est du Bassin parisien, entre PAC 92 et Agenda 2000
}

Sustainable agriculture in a system of large-scale cultivation: the example of plateaus in the south-eastern part of the Paris Basin, between CAP 92 and Agenda 2000

Dauerhafte landwirtschaftliche Entwicklung in Gebieten mit grossen Anbauflächen : Die Ebenen im Südosten Pariser Beckens zwischen Gemeinsamer Agrarpolitik 1992 und Agenda 2000

\section{Geneviève Pierre}

\section{(2) OpenEdition}

Édition électronique

URL : http://journals.openedition.org/rge/2526

DOI : $10.4000 /$ rge.2526

ISSN : $2108-6478$

Éditeur

Association des géographes de l'Est

Édition imprimée

Date de publication : 1 juin 2002

ISSN : 0035-3213

\section{Référence électronique}

Geneviève Pierre, «L'agriculture durable en système de grandes cultures : l'exemple des plateaux du sud-est du Bassin parisien, entre PAC 92 et Agenda 2000 », Revue Géographique de l'Est [En ligne], vol. 42 / 3 | 2002, mis en ligne le 09 décembre 2010, consulté le 08 septembre 2020. URL : http:// journals.openedition.org/rge/2526; DOI : https://doi.org/10.4000/rge.2526

Ce document a été généré automatiquement le 8 septembre 2020.

Tous droits réservés 


\title{
L'agriculture durable en système de grandes cultures : l'exemple des plateaux du sud-est du Bassin parisien, entre PAC 92 et Agenda 2000
}

\author{
Sustainable agriculture in a system of large-scale cultivation : the example of \\ plateaus in the south-eastern part of the Paris Basin, between CAP 92 and \\ Agenda 2000 \\ Dauerhafte landwirtschaftliche Entwicklung in Gebieten mit grossen \\ Anbauflächen : Die Ebenen im Südosten Pariser Beckens zwischen Gemeinsamer \\ Agrarpolitik 1992 und Agenda 2000
}

Geneviève Pierre

\section{Introduction}

1 Les plateaux du sud-est du Bassin parisien, plateau de Bourgogne du département de l'Yonne, plateau de Langres en Côte-d'Or et Haute-Marne et plateau du Barrois hautmarnais, appartiennent aux régions agricoles de grande culture les plus concernées par la réforme de la PAC de 1992 et l'Agenda 2000 (G. Pierre, 2001). Ces plateaux présentent des particularités agronomiques qui les distinguent des régions de grande culture à hauts rendements du centre du Bassin parisien (Colloque "petites terres ", 1985). Sur les auréoles calcaires du Jurassique des marges sud et est du Bassin parisien, des terres à cailloux ou "petites terres" s'étendent, depuis le Barrois meusien jusqu'à la Champagne du Berry et aux terres de groies du Poitou-Charentes (figure 1). Les plateaux de Bourgogne, de Langres et du Barrois de la Haute-Marne (figure 2) présentent les différentes nuances de spécialisations de l'ensemble des terres à 
cailloux, depuis l'hyperspécialisation en grandes cultures (blé, orge et colza uniquement) jusqu'aux systèmes mixtes grandes cultures - herbivores. Le faible potentiel de rendement par hectare de SAU a été compensé, depuis 30 ans, par une logique forte d'agrandissement de la superficie cultivée par exploitant, d'augmentation de la productivité, autour de quelques productions agricoles rarement valorisées sur place, dégageant une très faible valeur ajoutée "locale", alors que des alternatives culturales sont difficiles à définir. La logique de la PAC, par les paiements compensatoires à l'hectare de SCOP (surfaces en céréales, oléagineux et protéagineux), a été favorable à l'évolution de leurs revenus, tout en donnant une valeur administrative à un espace agronomique médiocre par l'importance des terres primées. Or, n'a-t-elle pas renforcé le modèle unique de développement fortement consommateur de foncier, sans que des alternatives aient été envisagées? Les hauts revenus garantis n'ont-ils pas paradoxalement renforcé la dépendance de cet espace de production à des décisions extérieures (politique européenne) sur lesquelles il a peu de prise, dans un contexte d'ouverture croissante à un marché mondial fluctuant et de plus en plus concurrentiel (Le Roy P., 1993) ? Comment définir la durabilité agricole de cet espace agricole, inséré dans des milieux ruraux souvent qualifiés de fragiles (plateau de Langres notamment) et pour lesquels l'activité agricole est incontournable dans le tissu économique, condition première d'un développement durable? Cette forme d'agriculture ayant à la fois surinvesti et radicalement simplifié le paysage, peutelle être articulée avec des logiques de développement local territorial ?

Figure 1 : Les « terres à cailloux » des marges sud et est du Bassin parisien

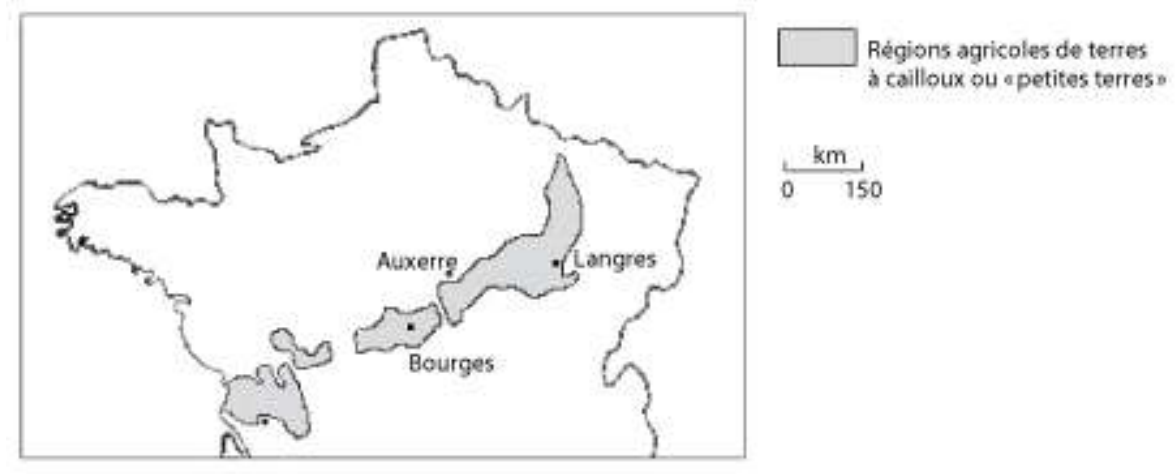

1a : Localisation en France, sur l'auréole jurassique du Bassin parisien 


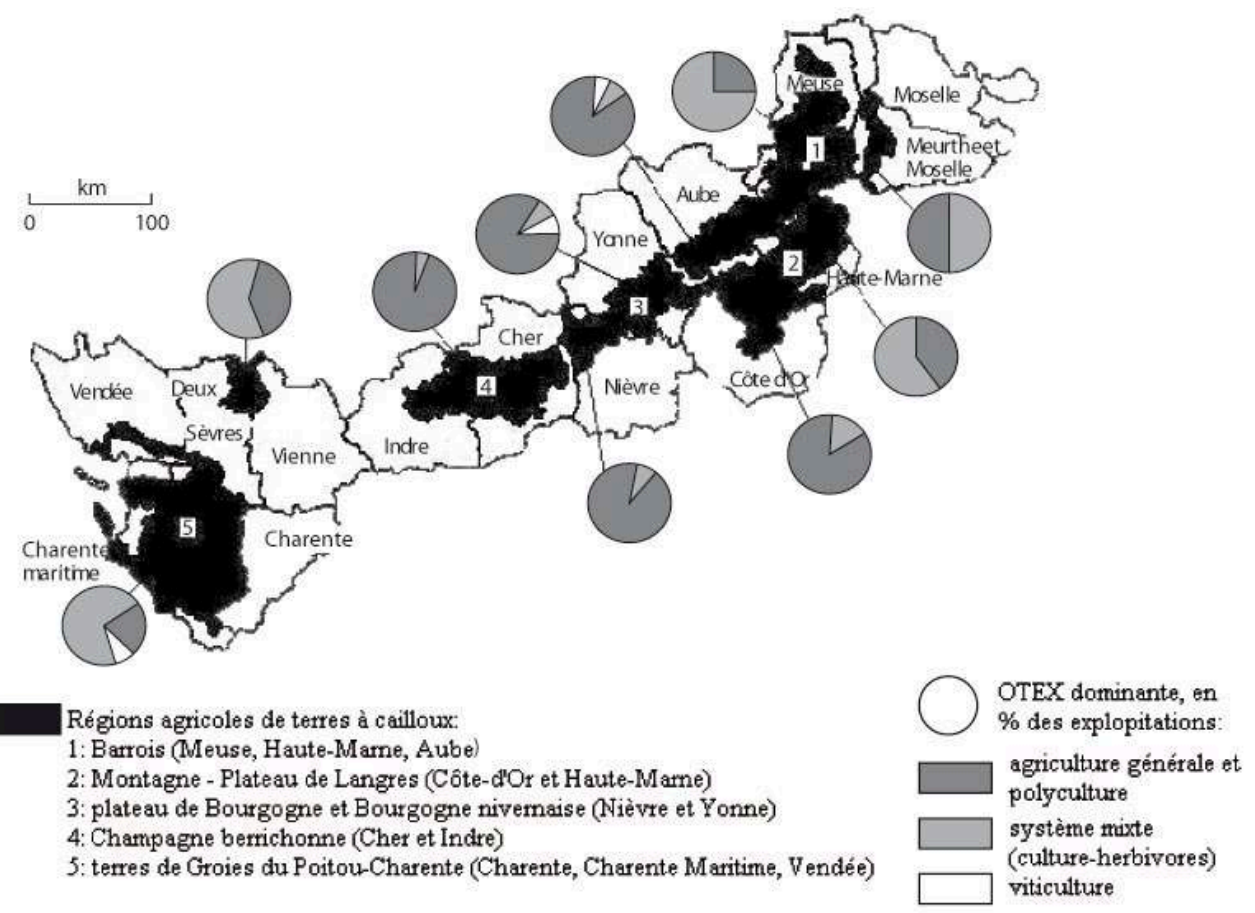

1b : Les petites régions agricoles des plateaux calcaires et de « terres à cailloux » du sud-est du Bassin parisien

Source : ITCF (institut technique des céréales et fourrages), 1985 et RGA 1988

Figure 2 : Les petites régions agricoles de plateaux des départements de la Côte-d'Or, de la HauteMarne et de l'Yonne

petite region agricole (PRA)

de "terres à cailloux"

ПIII autre PRA intégrée à la zone d'étude

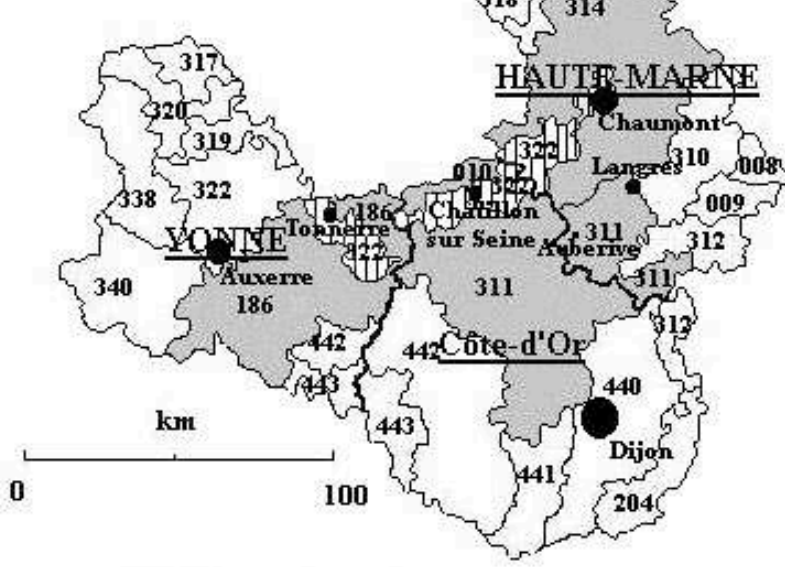

limites départementales
PRA selon le SCEES, RGA

(ministère de l'agriculture):

008: Apance

009: Amance

010: Tonnerrois

012: Vallage

186: Plateaux de Bourgogne

204: Val de Saône

310: Bassigny

311: Plateau langrois - Montagne

312: Vingeanue

314: Barrois

317: Chanpagne crayeuse

318: Chanpagne humide

319: Pays d'Othe

320: Basse Youne

321: Perthois

322: Vallées de l'Yonute à la Marne

338: Gâtinais pauvre

340: Puisaye

440: plaine viticole de Bourgogne

441: Côte viticole et arrière-côte

442: Autunois-Auxois-Terre plaine

443: Morvan 


\section{Une des principales conséquences de la PAC de 1992 : l'agrandissement accentué de structures d'exploitation déjà grandes}

\section{Le modèle de développement agricole des plateaux du sud-est du Bassin parisien s'est particulièrement bien adapté aux principales dispositions de la nouvelle PAC de 92 (Charvet J.P., 1997), notamment dans ses implications foncières}

2 Le système de compensation directe à l'hectare qui se substitue à l'ancien système où la quantité produite était valorisée par des prix garantis à la production, les premières mesures de préretraite agricole, le gel obligatoire des terres céréalières et oléagineuses (5 à $15 \%$ au cours des années 1990 en moyenne), les MAE (mesures agrienvironnementales) prônant des modèles d'extensification, la nécessité de mieux maitriser ses coûts de production (charges variables) et de réaliser des économies d'échelle (sur les charges de mécanisation par exemple), concourent à l'augmentation de la SAU par actif. Cette logique de développement est une tendance lourde des plateaux, depuis les années 1950-1960, à partir du moment où l'installation de la grande culture a permis la réutilisation par l'agriculture d'espaces laissés à la friche depuis plusieurs décennies, ainsi que le programme des « friches de l'est ", initié au début des années 1960, l'a montré (Cointat M., 1960). C'est, au départ, le vide démographique et la friche qui ont permis ce développement agricole, à l'instar de ce que Violette Rey a exposé pour la Champagne du Berry (Rey V., 1982) : un système de production adapté à un milieu agronomique médiocre, avec des rendements de 20 à 30 quintaux à l'hectare inférieurs, pour le blé, à ceux des systèmes de production hyper spécialisés du centre $\mathrm{du}$ Bassin parisien, et dans lequel les revenus par hectare de SAU (superficie agricole utile) sont faibles, alors que les revenus par UTA (unité de travail-année) sont beaucoup plus favorables: l'agrandissement est vu comme le moyen de compenser une faible valeur ajoutée par ha de SAU. Dans les années 1970-1980, l'agrandissement des exploitations s'amplifie, au moment où la spécialisation culturale s'affirme, autour du blé, de l'orge et du colza, seule tête d'assolement envisageable compte tenu des contraintes agronomiques, techniques et édaphiques (colloque "petites terres ", 1985). La part de l'élevage régresse nettement, même si elle se maintient dans les systèmes mixtes mieux représentés en Barrois et sur le plateau de Langres haut marnais que sur les plateaux bourguignons de l'Yonne.

Or, la PAC 92 a donné à cet espace de production les moyens d'accélérer les tendances lourdes du modèle traditionnel de développement (figure 3). 
Figure 3 : Le modèle dominant de développement agricole des plateaux du sud-est du Bassin parisien, face à la PAC de 1992 et à l'Agenda 2000

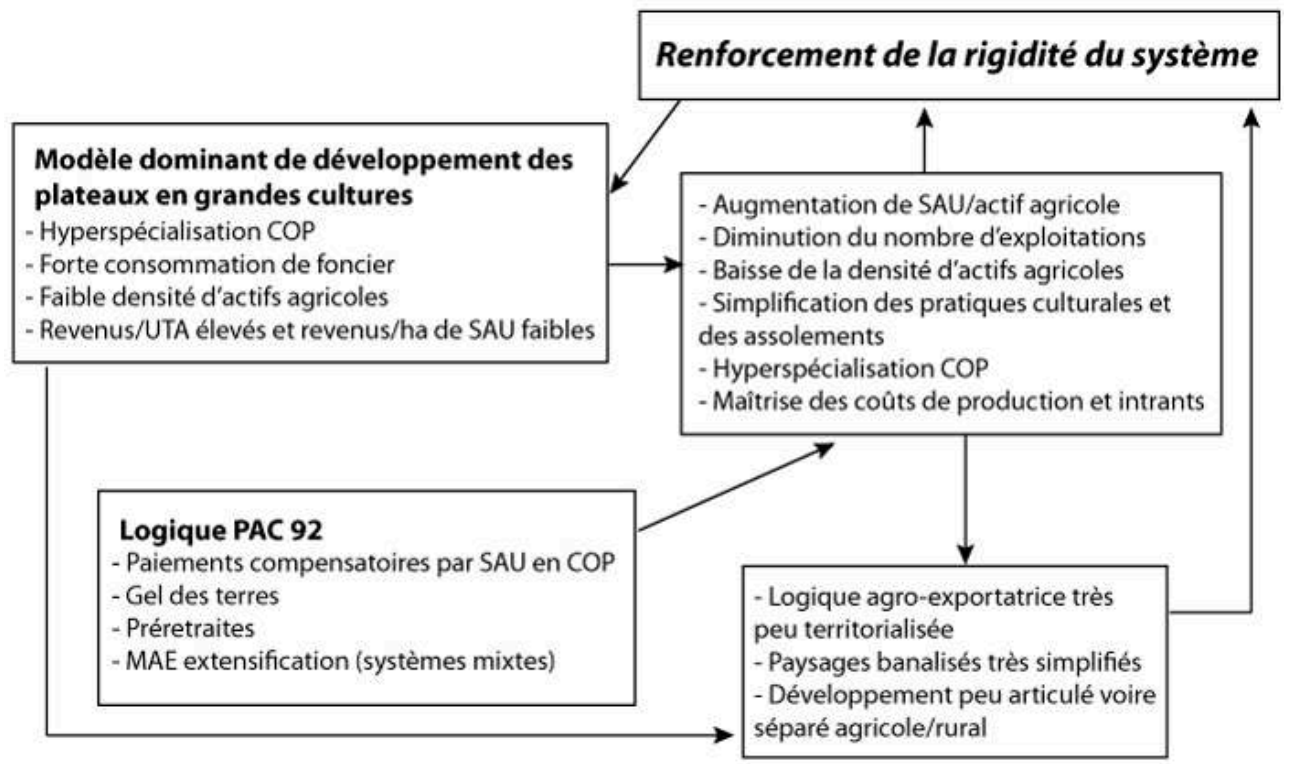

4 Dès les premières années, les départements des marges sud et est du Bassin parisien se distinguent par la taille moyenne élevée des exploitations agricoles : la Haute-Marne et l'Yonne apparaissent, en 1997, aux $2^{\mathrm{e}}$ et $4^{\mathrm{e}}$ rangs des départements français (97 et 91 hectares). La SAU moyenne par exploitation aurait augmenté de plus de $50 \%$ entre 1990 et 1997, en Haute-Marne. Cela fait généralement plus que compenser la perte de valeur ajoutée par hectare de SAU liée aux baisses de prix garantis. Cela est confirmé par les résultats du RGA (Recensement général de l'agriculture) 2000 : la Haute-Marne figure désormais en tête des départements français pour la SAU/exploitation professionnelle, avec 146 ha. On a atteint 135 hectares en Côte-d'Or, pour les exploitations professionnelles hors viticulture. Les exploitations de plus de 150 hectares occupent plus de $50 \%$ de la SAU dans l'Yonne, mais $25 \%$ et $61 \%$ de la SAU en Haute-Marne. Le nombre des exploitations de plus de 300 ha a été multiplié par 4 en Haute-Marne entre 1988 et 2000.

5 L'agrandissement, concentré sur les premières années de la réforme, se ralentit à partir de 1997-1998, comme le montrent les déclarations du fichier PAC-ACS (fichier d'aides compensatoires au gel des terres, disponible dans les DDA). Sur les plateaux de Bourgogne de l'Yonne, alors que la SAU moyenne par dossier PAC est de 113 hectares en 1993, elle a encore augmenté de $20 \%$ (la plus forte augmentation départementale) entre 1993 et 1997. Dans certains cantons du Barrois haut-marnais, la superficie moyenne par dossier PAC en 1994 est de 131 hectares (Poissons), voire 146 hectares (Saint-Blin). Sur la zone d'étude des plateaux (G. Pierre, 2001), la superficie moyenne par dossier PAC-ACS en 1997 est de 141 hectares ; elle s'élève à 175 hectares à AndelotBlancheville (Barrois haut-marnais), à l'Isle-sur-Serein (plateaux de Bourgogne de l'Yonne), ou à Grancey-le-Château (plateau de Langres, Côte-d'Or). Cela constitue des records en France.

6 La première mesure de préretraite agricole, mesure nationale d'accompagnement à la réforme de la PAC (1992-1994), combinée à un facteur primordial, la structure par âge de la population agricole (notamment la part des plus de 55 ans, directement concernés par la mesure de préretraite), constituent l'explication fondamentale. Cependant, la 
mesure de préretraite s'est appliquée dans un cadre national, sans restrictions géographiques ou techniques, pour encourager l'agrandissement des exploitations en zones difficiles ou défavorisées, ce qui ne correspond pas à l'agriculture des plateaux. L'indicateur démographique apparait primordial puisque la préretraite peut avoir un impact fort là où le pourcentage des plus de 55 ans, le public concerné par la mesure, est élevé. Or, ce pourcentage est supérieur à $39 \%$ sur les plateaux, en 1988, selon le RGA; le pourcentage des plus de 50 ans s'élevant même à $53 \%$. Ainsi, l'effet incitateur de la préretraite, renforcé par le poids élevé des exploitants âgés, a-t-il eu des répercussions foncières importantes, par le volant notable de terres à attribuer. Cependant, c'est dans les plateaux icaunais que cette mesure a eu le moins d'impact, là où, pourtant, le pourcentage d'augmentation de la SAU moyenne par dossier a été le plus important; il faut tenir compte des départs « directs » en retraite.

Les terres libérées ont essentiellement servi à l'agrandissement d'exploitations existantes. Une enquête menée par le CNASEA (Centre National pour l'Aménagement des Structures d'Exploitation Agricoles) Lorraine-Champagne-Alsace, sur un échantillon de $20 \%$ des repreneurs de terres libérées par la préretraite en HauteMarne, entre 1992 et 1994, montre que $40 \%$ des enquêtés avancent un besoin d'agrandissement, dans un département où la taille moyenne des exploitations est déjà très élevée. Depuis la loi de modernisation de l'agriculture de 1995, la réglementation de la préretraite privilégie l'installation de jeunes agriculteurs. Toutefois, le nombre de DJA (dotations aux jeunes agriculteurs) sur les plateaux marque, entre 1995 et 1999, un net recul: leur nombre est passé de 101 à 58 (il est même divisé par trois sur les plateaux de Bourgogne de l'Yonne). Cependant, si le renouvellement des exploitants a en partie eu lieu, il aboutit aujourd'hui à une situation de quasi blocage foncier, la structure par âge ayant été fortement rajeunie : les moins de 50 ans représentent, selon le RGA de 2000, plus de $55 \%$ des exploitants contre environ $45 \%$ en 1988. Cela peut fournir un élément d'explication au faible nombre de DJA enregistré depuis la fin des années 1990. D'autre part, l'inévitable pression foncière qui en résulte (la part importante des terres primées dans la SAU donne une valeur administrative à la terre), ainsi que la taille et la dimension économique élevées des exploitations actuelles peuvent décourager les candidats à la reprise. La tension sur le marché foncier est perceptible, là où les transferts fonciers ont été les plus intenses dans les années 1990, et risque de durer jusqu'en 2005-2010. Si le prix moyen des terres reste modeste en grandes cultures, les prix maxima relevés lors des échanges ont eu tendance à s'envoler, avec des hectares négociés à plus de 30000 francs, sur les plateaux du Barrois ou de Bourgogne (jusqu'à $47000 \mathrm{~F} / \mathrm{ha}$ en 1998), selon les SAFER (Sociétés d'Aménagement Foncier et d'Etablissement Rural).

8 Dans ce cas, existe-t-il un risque de déprise par impossibilité économique de la reprise de l'exploitation?

\section{Le développement de l'agriculture de groupe, à travers des formes sociétaires nouvelles ou plus traditionnelles, définit des solutions nouvelles pour l'occupation et l'utilisation du territoire agricole}

9 On a connu un développement considérable, ces dernières années, des formes sociétaires d'exploitation, parfois dans le cadre de définitions juridiques nouvelles : si les GAEC (Groupement Agricole d'Exploitation en Commun) ne sont pas nouveaux, le 
développement des SCEA (Société Civile d'Exploitation Agricole) et surtout des EARL (Entreprise Agricole à Responsabilité Limitée), à la réglementation plus souple que le GAEC, représente une tendance récente. Or cela peut avoir trois conséquences importantes : minimiser la SAU (Superficie Agricole Utile) cultivée par exploitant (bien que les EARL puissent être unipersonnelles), favoriser l'installation de jeunes agriculteurs, en remplacement d'un associé, et définir des solutions nouvelles à la pérennité d'exploitations de plus en plus grandes, tant par leur superficie que par leur dimension économique. Le nombre des sociétés augmente dans les années 1990 alors que l'effectif des déclarants PAC diminue. En Haute-Marne, en 1997, les exploitations sociétaires des plateaux du Barrois occupent $53 \%$ de la SAU, essentiellement en GAEC. Sur les plateaux de Haute-Marne, en 1997, les superficies par dossier sont de 144 hectares, mais de 104 hectares «seulement " par exploitation non sociétaire (dans le canton d'Andelot-Blancheville, 175 et 116,5 hectares).

10 Aussi, une augmentation de superficie peut être le préalable ou le corollaire à l'installation d'un nouvel associé. Alors que la superficie moyenne à l'installation pour un jeune agriculteur est élevée sur les plateaux de Bourgogne de l'Yonne (75 à 100 hectares selon les fichiers ADASEA (Association Départementale pour l'Aménagement des Structures d'Exploitation Agricole), dans les années 1990, elle est inférieure en Haute-Marne (50 à 70 hectares en Barrois, cantons de Blaiserives, Doulaincourt, Chaumont, Arc-en-Barrois), là où le pourcentage d'installations sociétaires (GAEC familiaux) est le plus important (64\% en nord Barrois). Dans l'Yonne, le pourcentage d'installations en sociétés est bien plus faible, mais se réalise sous la forme d'EARL (dont beaucoup sont unipersonnelles) qui sont devenues majoritaires en 1999, à l'instar des plateaux de Côte d'Or. Enfin, l'installation d'un jeune agriculteur n'impose pas toujours une acquisition foncière préalable en société, compte tenu des superficies moyennes élevées des exploitations: en 1996-1997 (ADASEA de Haute-Marne), dans $40 \%$ des cas, l'installation d'un jeune est l'occasion de créer un GAEC.

\section{Les stratégies de maintien ou de consolidation du revenu}

11 Dans ce milieu agronomique, à rendement limité, et face à un marché davantage mondialisé, mettant les différentes régions de grande culture en concurrence (Le Roy P., 1993), les stratégies de maintien ou de consolidation du revenu doivent être diversifiées.

12 L'agrandissement des exploitations a un rôle à jouer, mais la maîtrise accrue des coûts de production devient aussi la condition de la durabilité ; cela passe par l'agriculture raisonnée, le contrôle des charges variables, la traçabilité, une politique de qualité, de contractualisation, auprès des organismes stockeurs.

\section{Le poids important du foncier dans l'évolution des revenus au cours des années 1990.}

La dimension structurelle joue un rôle majeur dans la progression plus favorable des revenus, au cours des années 1990, sur les plateaux par rapport à d'autres régions de grandes cultures céréalières qui n'ont pas connu une évolution structurelle d'une telle 
ampleur (Agreste, SCEES, RICA 1993, 1996, 1998). En rendant une partie des aides publiques indépendantes des résultats de production, mais en privilégiant les aides assises sur la SCOP, on a renforcé le poids du foncier dans la formation des revenus. Les différences de revenus agricoles résultent de moins en moins des performances économiques des exploitations et de plus en plus des mécanismes de redistribution publique (Butault J.B., Lerouvillois Ph., 1999). L'exemple du département de l'Yonne illustre bien cette différence dans l'évolution des revenus, entre régions agricoles de grande culture «à fort potentiel » (le nord du département, Gâtinais, Basse-Yonne et Champagne crayeuse) et les régions «à faible potentiel » que constituent les plateaux, où l'agrandissement des exploitations a été plus marqué. L'évolution du revenu agricole par UTH (unité de travail humain), de 1991 à 1997, a été plus favorable, de 40000 francs en moyenne, par an, à partir de 1994, sur les plateaux à faible potentiel que dans les régions céréalières du nord du département (centre d'économie rurale de l'Yonne, 1996, 1997 et 1998 et Detaille J.C., Observatoire départemental de l'Yonne, 1991-1995). C'est à partir de 1993 que le "retournement de situation" s'est opéré, même si le produit agricole par hectare de SAU reste à l'avantage du nord du département (Observatoire de l'agriculture bourguignonne, juillet 1996, juillet 1997, juillet 1998).

Cependant, même entre régions de plateaux, on observe des différences qui tiennent au degré de spécialisation en grandes cultures plus ou moins marqué des systèmes de production. Ceci est illustré par le département de la Haute-Marne entre le «Barrois centre» (incluant en partie la région agricole de la "vallée») et la "grande Montagne » haut-marnaise, c'est-à-dire le plateau de Langres central (Auberive) : c'est ici que le nombre d'UTH par exploitation est le plus élevé et que l'agrandissement de la SAU a été le moins prononcé : on a atteint des limites de productivité soumises à la forte représentation de l'élevage bovin laitier en système mixte. En "grande Montagne ", le produit agricole par hectare de SAU ne représente que $80 \%$ de celui du Barrois centre en 1998, en système mixte grandes cultures - herbivores; il n'a augmenté que de 6,8\% entre 1993 et 1998 en " grande Montagne» contre 10,3\% en Barrois centre! La marge brute par hectare de SAU évolue de façon moins favorable que dans les autres régions de plateaux et ne dépasse guère $4000 \mathrm{~F} /$ ha depuis 1991 , alors qu'elle atteignait près de $5000 \mathrm{~F} /$ ha de SAU sur les plateaux de Bourgogne de l'Yonne et en Barrois central, en 1998.

\section{Une productivité accrue du travail agricole, dans des systèmes de culture de plus en plus spécialisés.}

15 Un élément fondamental de la définition des revenus est le résultat de la vente des productions agricoles, par rapport aux coûts de production, se traduisant, sur les plateaux, par une simplification des pratiques culturales et des assolements adaptés à une forte productivité du travail agricole, autour de productions primées par la PAC: blé, orge et colza.

16 Les productions céréalières et oléagineuses se sont de plus en plus affirmées dans les années 1990 (fichiers PAC). Si les SCOP représentent 77,6 \% de la SAU sur l'ensemble des plateaux, elles occupent plus de $85 \%$ de la SAU sur les plateaux icaunais, $77 \%$ sur les plateaux de Côte-d'Or et $70 \%$ sur ceux de Haute-Marne, où la part des cultures fourragères et prairies artificielles est plus élevée qu'ailleurs (plus de $15 \%$ de la SAU sur le plateau de Langres), alors que les STH occupent toujours une part non 
négligeable de la SAU : $21 \%$ dans le canton d'Auberive, 26,6\% dans celui de Prauthoy (plateau de Langres haut-marnais), au milieu des années 1990. Cependant, les SCOP se sont affirmées depuis 1992; les superficies fourragères ont reculé, notamment en Barrois et on a même connu un « grignotage » des STH par les SCOP avant 1992, pour en faire des terres primables.

C'est le blé tendre qui a le plus profité de cette évolution (40 à $45 \%$ de la SAU sur les plateaux de Bourgogne de l'Yonne), mais le colza, seule tête d'assolement, a aussi accru ses superficies. Selon le dernier RGA, les surfaces en blé ont augmenté de $12 \%$ dans l'Yonne à $30 \%$ en Haute-Marne, au détriment de l'orge, alors que le colza a encore progressé dans des espaces déjà hyper spécialisés dans cette production, pour atteindre un maximum de $30 \%$ des terres labourables en système de grandes cultures. Dans le canton d'Auberive (plateau de Langres central), le blé occupe $43 \%$ des surfaces céréalières et l'orge (production pouvant servir à l'élevage), $48 \%$. Dans le canton de Juzennecourt (plateau du Barrois), le blé et l'orge utilisent respectivement $57 \%$ et $34 \%$ des superficies céréalières. Aucune production «de repli " n'a été envisagée: la diversité culturale est difficile à définir sur les plateaux, compte tenu de la nature du terrain constituée de petites terres à cailloux, aux sols minces à faible réserve hydrique, dans lesquelles les pommes de terres, les protéagineux, le tournesol, le maïs et la betterave sont difficilement envisageables.

Les assolements sont de plus en plus raisonnés en fonction de l'évolution des cours, notamment par la place faite au colza. La part de l'orge se maintient mieux en système mixte (plateau de Langres notamment) mais les agriculteurs rencontrés à la fin des années 1990 (G. Pierre, 2001) prévoient de revoir à la baisse la part du colza, en fonction de l'évolution des cours (en baisse à la fin des années 1990) et des dispositions de l'Agenda 2000, bien que cela soit la seule tête d'assolement possible. Seul le blé semble durablement s'affirmer, au risque d'un développement mono- cultural. L'assolement type devient de plus en plus colza-blé-blé-orge.

\section{Les contraintes et facteurs de commercialisation des quelques matières premières agricoles produites sur les plateaux : vers la reconnaissance d'un produit de qualité ?}

Ces matières premières, non valorisées sur place, sont vendues hors de la région avec une contrainte de transport due à l'éloignement maximal des principaux ports et grandes voies fluviales. Cependant, la Bourgogne est la région céréalière excédentaire la plus proche de l'Italie du nord: $50 \%$ des blés de la coopérative 110 Bourgogne sont destinés à ce marché. L'ouverture au marché mondial (Charvet J.P., 1996), à partir des zones portuaires, renforce le poids du transport dans la définition des prix payés au producteur, qui pèse d'autant plus que le cours des céréales est bas : il faut davantage rationaliser le stockage et l'expédition. Parallèlement, dans les années 1990, le rapport producteur-collecteur-transformateur s'est inversé. Il ne suffit plus de proposer des marchandises en qualité standard pour être certain de les vendre. Le marché est devenu plus exigeant et plus pointu. Or, le blé produit sur les plateaux de Bourgogne, de Langres et du Barrois, du fait de la faiblesse des rendements, présente un taux de protéine plus élevé ( $12 \%$, voire plus) que dans d'autres régions agricoles comme la Champagne crayeuse $(10,5 \%)$. Cette bonne qualité boulangère est un atout sur le marché italien. C'est ce que les collecteurs cherchent à valoriser, en contractualisant la 
production, afin de fournir des lots de qualité homogène, répondant parallèlement à des normes sanitaires et techniques, de traçabilité du produit, ainsi qu'à une rémunération en fonction $\mathrm{du}$ taux de protéine, généralisée assez tôt chez les organismes stockeurs des plateaux. La mise en place des contrats meuniers permet, tout en valorisant la qualité produite par un tarif majoré auprès du producteur, de mieux orienter l'offre. Si, selon l'AGPB (Association Générale des Producteurs de Blé), en 1999, les productions céréalières sous contrat représentent $10 \%$ de la collecte céréalière en moyenne, en France, les agriculteurs des plateaux sont en avance : à la coopérative Dijon Céréales, 60 \% des agriculteurs du Châtillonnais (nord de la Côte d'Or) sont contractants, pour 35 à $40 \%$ de la collecte céréalière. A 110 Bourgogne (plateaux de l'Yonne), les contrats meuniers sur le blé et l'orge représentent, en 1999, 50 \% de la collecte. On peut se spécialiser également dans des "niches de production" très particulières et contractualisées : qualités spécifiques de production avec respect de normes sanitaires précises et rigoureuses pour le marché de l'alimentation pour bébés, par exemple. Le stockage privé (chez le producteur), encouragé pour les contrats meuniers, est également valorisé par la coopérative (à Dijon Céréales, la prime de stockage est de $30 \mathrm{~F} /$ tonne en 1999-2000). Parallèlement, pour maintenir leur revenu, les agriculteurs ont modifié leurs pratiques de commercialisation, en contrôlant, par l'intermédiaire de leur organisme stockeur, le moment de la mise en vente de leur production, en fonction de l'évolution des cours: c'est la mise en dépôt, avec majoration à partir du prix moyen de campagne défini par la coopérative.

\section{Le maintien de la marge brute par le contrôle des charges de production}

Dans une stratégie ancienne, mais accentuée par la PAC de 1992, de contrôler les charges de production et maintenir la marge brute/ha, les agriculteurs ont développé des économies d'intrants, conformes aux discours et aux pratiques de l'agriculture raisonnée, avant même que cela soit devenu un argument de promotion et de vente à la mode (Sébillotte M., 1996).

21 Si on ne constate pas de volonté durable de diminution des charges par hectare de SAU (Centre d'Economie rurale de Bourgogne et Centre de Comptabilité et d'Economie Rurale de Haute-Marne), les charges d'intrants sont en moyenne de $25 \%$ inférieures à celles des régions de grandes cultures du nord de l'Yonne (Basse-Yonne, Champagne crayeuse). Les services agronomiques des Chambres d'Agriculture, dès les années 1980, relayés par les conseils techniques des organismes stockeurs dans les années 1990, ont privilégié la consommation raisonnée des engrais, par une meilleure connaissance du terrain, des besoins de la plante et des capacités limitées de rendement sur les terres à cailloux : on a diminué la fertilisation azotée de 10 unités/ha, pour la porter à $170-180 \mathrm{~kg} / \mathrm{ha}$ sur les plateaux, alors qu'en Champagne crayeuse, on dépasse souvent 200 unités. Les doses d'engrais de fond ont davantage diminué que l'azote qui joue un rôle majeur dans la détermination du rendement. Ainsi, la directive européenne "nitrates », mise en place en décembre 1991 pour contrôler la pollution due aux nitrates d'origine agricole, et qui doit s'appliquer sur les zones vulnérables dont font partie les plateaux calcaires du sudest du Bassin parisien, s'avère en fait très peu contraignante, puisqu'on se situe, sur les plateaux, en dessous des normes préconisées $(210 \mathrm{Kg} / \mathrm{ha} / \mathrm{an})$ dans le plan défini jusqu'en 
1999. Pour les années 2000 à 2003, la norme, révisée à la baisse, (jusqu'à $170 \mathrm{~kg} / \mathrm{ha} / \mathrm{an}$ ) est assez proche de ce qui se pratique sur les plateaux.

Seules les charges de mécanisation ont connu une augmentation, l'introduction de revenus fixes par les primes étant l'occasion de renouveler ou enrichir le parc de matériel et surtout de l'adapter à des exploitations agrandies, dans le cadre de stratégies individuelles, puisque le recours aux ETA (entreprises de travaux agricoles) ainsi qu'aux CUMA (coopératives d'utilisation du matériel en commun) est marginal dans les systèmes de grandes cultures les plus spécialisés, par individualisme, mais aussi à cause des limites techniques : tous les exploitants ont besoin du même matériel, au même moment, sur de vastes superficies de production. Sur les plateaux hautmarnais, là où les systèmes mixtes sont dominants, imposant une diversité de matériels, les CUMA ont trouvé un écho plus favorable. La création de cercles d'échange du matériel agricole en 1991, fonctionnant surtout en Barrois et pour le matériel de culture, entre agriculteurs disposant d'un surcroît de matériel et exploitants demandeurs de travaux agricoles, permet de diminuer les charges de mécanisation ou la somme de travail en période de pointe, mais cela ne touche qu'à peine $10 \%$ des agriculteurs en 1999, sauf dans le canton de Châteauvillain, où le taux de pénétration atteint $20 \%$.

Le matériel doit également satisfaire les exigences d'exploitations de plus en plus grandes, dans une logique d'augmentation de la productivité. Les agriculteurs travaillant sur ces plateaux ont été les plus enclins à développer les techniques de semis simplifié, de non labour, voire, plus rarement, de semis direct. La remise en cause du labour s'est, depuis les années 1990, nettement généralisée, sans perte de rendement, plus sous la forme du «labour minimum » que du non labour total. Les techniques «sans labour » permettent un travail du sol à 10-15 cm de profondeur, par des outils à dents, à disques ou vibroculteurs, et évitent de faire remonter les cailloux, tout en atténuant l'usure accélérée du matériel constatée dans ce type de sol; elles autorisent un gain de temps considérable et sont adaptées à l'extension de la superficie cultivable par exploitant et aux pointes de travail en système hyper- spécialisé. Le semis simplifié n'impose pas de changer le parc de matériel; en revanche, le semis direct s'est peu développé car nécessite un renouvellement complet, puisqu'un seul matériel est nécessaire : système « Horsch » ou « Dutzi » (noms des marques fabriquant ce type de matériel), avec un tracteur très puissant. Ces techniques culturales, étudiées au cours des années 1990 dans un souci de limitation des charges de mécanisation et donc des coûts de production, répondent en fait davantage à des stratégies d'augmentation de la productivité du travail.

De plus, là comme dans les pratiques de contrôle des intrants, les objectifs économiques l'emportent nettement, dans l'esprit des agriculteurs, sur les bénéfices environnementaux. Les MAE (Mesures agri-environnementales) dont certaines ont pu concerner les plateaux (périmètres très limités de protection des eaux, rares conversions à l'agriculture biologique, jachère faunistique liée aux activités cynégétiques, opération locale " plateaux de Bourgogne » de préservation de la flore) ont trouvé un écho marginal, essentiellement motivé, quand il a existé, par des impératifs de gestion, de façon souvent plus marquée que dans d'autres régions de grandes cultures (Fruit J.P. et Lompech M., 1997). 


\section{Un modèle extensif ou en voie d'extensification?}

Sur les plateaux, on ne cherche plus à tout prix à " pousser le rendement ». Le système de production apparaît de ce fait nettement moins intensif, en grande culture, qu'au centre du Bassin parisien. Cependant, on ne peut guère parler clairement de pratiques agricoles extensives ou d'extensification, malgré l'augmentation rapide de la dimension structurelle des exploitations compensant une perte de revenus par ha de SAU. (Béranger C., 1990 ; Guy P., Fraval A., 1998). Il n'y pas, sur les plateaux, de volonté de diminution parallèle des charges et du produit agricole/ha de SAU, par une maitrise des rendements. Le système de production est très productiviste, associant une intensification du facteur terre (par agrandissement, généralement en fermage) à une productivité élevée du travail agricole : il n'y a pas contradiction entre une logique extensive d'occupation de l'espace et une forte productivité du système de production. Cependant, on est encore loin du modèle américain en grande culture, même si la logique d'agrandissement à tout prix, avec une productivité de plus en plus élevée en monoproduction, peut y faire penser. On reste, sur les plateaux, dans une logique de rendements bien plus élevés que ceux que l'on connaît dans les "greniers à blé " (Charvet, 1985) américains et la taille moyenne des exploitations agricoles, bien qu'importante pour la France, reste modeste par rapport à la situation américaine. Si des craintes pour la transmission des exploitations peuvent apparaitre dans les années qui viennent, compte tenu de la lourdeur de la reprise, la volonté d'agrandissement des exploitants en place, dans des exploitations aux statuts juridiques nouveaux, éloigne tout risque de déprise de l'espace de production, sauf sur des superficies marginales, mal situées, éloignées. Toutefois, la mise en place d'un système de production s'engageant dans la voie de l'extensification avec maintien, voire augmentation du revenu agricole, ne peut qu'accentuer le modèle unique de développement par agrandissement soumis sur place aux possibilités de libérations foncières : or, jusqu'en 2005-2010, on connaît une situation de blocage foncier, ralentissant à court terme le développement général d'un modèle extensif de production. La question de l'extensification révèle certaines contradictions fondamentales des politiques agricoles, notamment en grande culture. L'extensification, considérée comme bonne pour l'environnement car jugée moins prédatrice pour le milieu, est indirectement dévalorisée dans le discours sur le développement durable du fait de ses conséquences, jugées négatives, sur les systèmes de production en grande culture : la diminution très nette du nombre d'exploitations, la très faible densité d'actifs agricoles, la simplification des paysages et des systèmes de culture liée à l'hyper spécialisation en blé, orge et colza et l'articulation rendue de plus en plus difficile entre développement agricole et développement rural.

\section{Quelles durabilités pour l'agriculture et les espaces ruraux?}

\section{Une agriculture fortement subventionnée et dépendante de décisions extérieures}

La modification de certaines pratiques agricoles, dans un système de production dont la logique de développement reste inchangée, ne règle pas le problème de la dépendance 
du revenu par rapport à l'extérieur (PAC et marchés mondiaux), qui est pourtant devenue la condition de l'apparente solidité du système et donc de sa durabilité.

Sur les plateaux de Bourgogne de l'Yonne, le poids des subventions dans l'EBE (excédent brut d'exploitation) est un peu plus élevé (92,5\% en 1996, selon le CER de Bourgogne) que dans les régions céréalières du nord du département. Sur les plateaux haut-marnais du Barrois et de Langres, cette dépendance par rapport à la PAC de 1992 apparaît un peu moins forte, compte tenu du poids de l'élevage en systèmes mixtes ( $75 \%$ de l'EBE et $27 \%$ du produit agricole), en 1996, selon le CCER de Haute-Marne, mais pour des productions soumises à d'autres aides (élevage bovin lait et viande).

Si la bonne tenue des cours des productions au cours des années 1990 a pu masquer les effets de cette dépendance, celle-ci se révèle brutalement à la fin des années 1990. La baisse des cours mondiaux du colza à la fin des années 1990, le nivellement du paiement compensatoire oléagineux sur celui des céréales par la réforme de la PAC 2000 et la modulation des aides directes au revenu décidées par la France dans la loi d'orientation agricole de 1999 afin de financer les CTE, touchent en priorité les exploitants des plateaux. La diminution des revenus par hectare de SAU encourage à s'agrandir, y compris en acquérant les entreprises agricoles les plus fragiles, à la dimension économique et structurelle trop faible pour subsister. Cette situation sociale difficile peut même atténuer à court terme les facteurs démographiques du «blocage foncier » actuel déjà évoqué.

\section{Une agriculture pérenne dans un milieu rural fragile?}

Le modèle de développement agricole des plateaux peut-il être infléchi alors que la PAC de 1992 a accentué son extrême rigidité, ne lui laissant que très peu de marges de manœuvre? Peut-on définir sa durabilité en dehors de l'efficacité économique et des hauts revenus dégagés qui suggèrent le maintien du modèle de développement par agrandissement? Comment ce système peut-il s'inscrire dans le débat actuel sur l'articulation développement rural/développement agricole pour définir une « campagne vivante » (Croix N., 2000), dans la perspective d'un développement rural durable, dont les CTE (contrats territoriaux d'exploitation), selon la loi d'orientation agricole française de 1999, constituent une des armatures? Par l'accord de Berlin (1999), le développement rural doit être le second pilier de la réforme de la PAC 2000. Or, sur les plateaux du sud-est du Bassin parisien, des trois fonctions reconnues à l'agriculture, une seule a été privilégiée, la fonction productive, qui utilise le plus possible l'espace: les pratiques agricoles ont entraîné la banalisation extrême des paysages, autour de parcelles de cultures de plus de 100 hectares, et de la forêt, très présente également. Cela définit un exemple particulier du rapport économie, environnement et société, visible entre autres à l'occasion des remembrements (Marochini E., 1999), mais laisse, sur les plateaux, peu de points d'appui autres que cynégétiques au développement du tourisme rural. Ainsi, la fonction "services" de l'agriculture a-t-elle été négligée.

Parallèlement, la PAC de 1992 a accentué le décalage entre une apparente prospérité agricole et une fragilité rurale réelle, notamment sur le plateau de Langres, mais surtout entre les logiques de développement rural, territorial, et la logique de filière, verticale, du développement agricole. Peut-on exclure l'agriculture des plateaux, et, de façon générale, la grande culture, de toute perspective de développement durable? Pour certains, le 
productivisme peut être amélioré par l'éco-conditionnalité et évoluer graduellement vers une logique de développement durable (Briel B. et Vilain L., 1999). Cependant, comment la grande culture du sud-est du Bassin parisien peut-elle devenir multifonctionnelle et territorialisée, articulée à une logique territoriale de développement, dans des espaces ruraux qualifiés souvent de fragiles?

\section{Quelques exceptions au modèle de développement dominant, articulant mieux développement agricole et développement rural}

31 On peut définir des nuances au modèle dominant de développement agricole des plateaux, notamment en ce qui concerne la partie haut-marnaise du plateau de Langres central (secteurs d'Auberive et de Prauthoy) pour laquelle une agriculture davantage territorialisée, reposant sur une volonté un peu plus forte qu'ailleurs de développement rural intégré, a pu voir le jour. Cette politique, certes modeste, s'applique à l'espace rural le plus fragile des plateaux, ainsi qu'à la région agricole la plus marquée par les systèmes mixtes de production, dégageant des revenus par exploitation, par ha de SAU et par actif moins importants qu'ailleurs sur les plateaux, alors que le nombre d'UTH/exploitation est le plus élevé. La démarche multifonctionnelle de PDD (plan de développement durable) a été menée en 1994 autour de la question de la diversification agricole et autour de l'objectif d'un meilleur raisonnement sur l'utilisation des fumures azotées. La définition de deux labels AOC de production fromagère (Epoisses et Langres) permet de territorialiser une partie de la production agricole locale, confortée par la définition de CTE collectifs. Ces démarches sont prolongées aujourd'hui par la naissance d'un pays de Langres intégrant le plateau, par la création d'un parc naturel du plateau de Langres débordant sur la Côte-d'Or et s'appuyant sur la forêt, ses ressources cynégétiques, la pêche, les sentiers de randonnée, dans l'espace rural le plus "vide» des plateaux mais également le plus préservé des effets paysagers de la grande culture. Une même démarche de pays est à l'œuvre en Tonnerrois (dans les cantons d'Ancy-Le-Franc, Cruzy-Le-Châtel, Flogny-LaChapelle, Noyers et Tonnerre), autour de la renaissance viticole et du tourisme rural, mais ne s'appuie pas sur la même logique collective de développement, intégrant moins les agriculteurs (hors viticulture) à ses projets.

\section{Conclusions : ce modèle agricole de développement est-il durable?}

L'accentuation du modèle de développement par agrandissement ne doit pas faire craindre la déprise de l'espace de production : le recensement général de l'agriculture de l'année 2000 montre que la SAU a été maintenue depuis 1988. Pourtant, les évolutions agricoles actuelles, résultant de la dépendance par rapport à des décisions économiques et politiques «extérieures » (baisse des cours du colza, alignement des paiements compensatoires du colza sur ceux des céréales, modulation des aides directes) entraînant une diminution du revenu des agriculteurs des plateaux, le nombre d'exploitations risque de diminuer rapidement au profit des entreprises à plus forte dimension économique et structurelle, sans que l'espace agricole de production, ni même la capacité de production s'en trouvent affectées. D'autre part, bien que l'articulation développement rural - développement agricole soit difficile à définir sur 
les plateaux, on ne peut envisager le développement durable sans l'agriculture tant, dans cet espace rural, l'activité agricole, sur-représentée, constitue un élément de prospérité incontournable pour maintenir un tissu économique minimum, tout en utilisant une large partie de l'espace non forestier. Or la durabilité agricole passe ici essentiellement par le raisonnement économique de filière, privilégiant le modèle unique de développement par agrandissement permettant de minimiser les coûts de production, mais surtout de profiter au mieux des principales dispositions de la PAC de 1992, c'est-à-dire les paiements compensatoires à la surface de production en céréales et oléo-protéagineux, découplés de la production. Cette forme d'agriculture est très mal articulée avec les perspectives de développement local durable, alors que sans elle, il n'y a pas de développement durable envisageable dans la plupart de ces régions de plateaux. Ces contradictions, dans la volonté actuelle (Loi d'Orientation Agricole française de 1999, Agenda 2000) de développer une agriculture multifonctionnelle, semblent difficiles à résoudre. En fait, un aspect fondamental du développement durable doit être évoqué : à quelle échelle géographique doit-on définir le "territoire durable » et l'échelle des plateaux, caractérisés par leur relative homogénéité, est-elle pertinente? Aussi, au nom du développement durable, faut-il admettre des espaces monofonctionnels, simplement producteurs de quelques matières premières exportées (les espaces agricoles de grandes cultures aux paysages simplifiés et à l'économie très spécialisée), contrebalancés par ailleurs par des espaces de loisirs, de nature (espaces forestiers) et de tourisme, basés également sur une agriculture multifonctionnelle, (polyculture, polyculture-élevage, élevage extensif dans des paysages herbagers)... Dans ce cas, c'est à une échelle régionale plus large que l'on peut envisager le territoire durable. Par ailleurs, si le modèle agricole de développement des plateaux apparaît aujourd'hui durable car économiquement viable et prospère, sa dépendance par rapport à l'extérieur (politique européenne plus sévère, marché mondial de plus en plus concurrentiel, notamment pour le colza), est porteuse d'inquiétude pour sa pérennité, alors que la rigidité du système de production rend très difficile la recherche de solutions alternatives : une évolution « à l'américaine » n'est pas à exclure à terme.

\section{BIBLIOGRAPHIE}

BÉRANGER C. (dir) (1990). - «Extensification, l'évolution des réflexions », Comité national extensification-diversification, séminaire des 23 et 24 octobre 1990, Ministère de l'Agriculture et de la Pêche, 191 p.

Butault J.B., Lerouvillois Ph. (1999). - « La réforme de la PAC et l'inégalité des revenus agricoles dans l'Union européenne », Economie et Statistique, n³29-330, pp. 73-86.

BRIEL B., VILAIN L. (1999). - «Vers l'agriculture durable », Ministère de l'Agriculture et de la Pêche, La Bergerie Nationale, Educagri Editions, Dijon, 143 p.

CHARVET J.P. (1985). - «Les greniers du monde », Economica, Paris, 368 p. 
CHARVET J.P. (1996). - « Le marché mondial des céréales au début du troisième millénaire », Déméter, rapport, A. Colin, Paris, pp. 16-57.

CHARVET J.P. (1997). - « La France agricole dans son environnement européen et mondial », Liris, $2^{\mathrm{e}}$ édition, $191 \mathrm{p}$.

CoInTAT M. (1960). - « Terres incultes et taillis pauvres de l'est de la France », Revue de l'économie du centre-est, $\mathrm{n}^{\circ}$ 7, pp. 130-134.

Colloque « 24 heures des petites terres », Auxerre, juin 1985, climat, sol système d'exploitation Institut Technique des Céréales et des Fourrages (ITCF), 1985.

CrolX N. (dir) (2000). - «Des campagnes vivantes ? Un modèle pour l'Europe »: Mélanges en hommage au professeur Jean Renard, Nantes, 696 p.

DeTAILLE J.-C. (1996). - Observatoire départemental 1991-1995, CER de L’Yonne.

«Economie du paysage et agriculture », Bti n 11-12, janvier-février/mars-avril 1993, Ministère de l'agriculture et de la pêche, $128 \mathrm{p}$.

FRUIT J.P., LOMPECH M. (1997). - « Les politiques agri-environnementales dans l'espace français », L'Information géographique, $\mathrm{n}^{\circ}$ 2, A. Colin, Paris, pp. 65-74.

GuY P., Fraval A. (dir) (1998). - « L'extensification », Dossiers de l'environnement, n 16, INRA, Paris, $150 \mathrm{p}$.

LE ROY P. (1983). - «Les agricultures françaises face aux marchés mondiaux », A. Colin, Paris, $282 \mathrm{p}$.

MAROCHINI E. (1999). - « Les remembrements en Moselle, entre économie, environnement et société : essai de géographie rurale appliquée ». Thèse de géographie, Univ. Metz, 600 p.

Pierre G. (2001). - « Les plateaux du sud-est du Bassin parisien entre PAC 92 et Agenda 2000, plateaux de Bourgogne, de Langres et du Barrois de la HauteMarne ». Thèse de géographie, Univ. Paris X, 702 p.

Observatoire de l'agriculture bourguignonne, Conseil régional et CER Bourgogne, résultats 1996, juillet 1997 ; résultats 1997, juillet 1998.

REY V. (1982). - «Besoin de terre des agriculteurs », Economica, Paris, 389 p.

SÉBILLOTTE M. (1996). - «Les mondes de l'agriculture, une recherche pour demain », INRA éditions, Paris, $258 \mathrm{p}$.

Sources :

RGA (recensement général de l'agriculture) 1988 et 2000.

Agreste-SCEES : RICA (réseau d'information comptable agricole 1993, 1996-97-98-99. Enquêtes de structure 1990-93-97.

ADASEA (Associations départementales pour l'aménagement des structures d'exploitations agricoles) Côte-d'Or, Haute-Marne et Yonne.

Centres d'économie rurale : CER Bourgogne, Yonne et CCER (centre de comptabilité et d'économie rurale) de Haute-Marne.

Fichiers PAC-ACS, DDA Côte d'Or, Haute-Marne et Yonne. 
SAFER (Société d'aménagement foncier et d'établissement rural) Côte d'Or, Yonne et HauteMarne.

\section{RÉSUMÉS}

Les plateaux du sud-est du Bassin parisien ont été particulièrement concernés par la réforme de la PAC de 1992 et l'Agenda 2000, en raison de la simplicité de leurs assolements : blé, orge et colza. La logique de la PAC a été favorable à l'évolution des revenus, en donnant une valeur administrative à un espace agronomique de "terres à cailloux » aux rendements médiocres. Or, la PAC de 1992 s'applique à un système de développement agricole dans lequel l'augmentation à tout prix de la productivité est une règle, au sein d'exploitations sans cesse agrandies, avec une main-d'œuvre agricole réduite, autorisant bien peu de marge de manœuvre. Cet espace agricole apparemment prospère est devenu très dépendant, pour sa durabilité, de décisions politiques extérieures (PAC) et des cours de productions de plus en plus mondialisés, dans un système de production très peu territorialisé. Comment définir un développement durable dans ces espaces agricoles de grandes cultures, insérés dans des milieux ruraux souvent qualifiés de fragiles (plateau de Langres) et pour lesquels l'activité agricole est incontournable dans le tissu économique ? Le résultat économique, lié à la dépendance vis-à-vis de la PAC et condition de la durabilité actuelle, pose des questions quant à la pérennité du modèle. Cette forme d'agriculture, ayant radicalement simplifié le paysage, s'articule mal avec des logiques de développement local territorial, malgré quelques rares contre-exemples.

The plateaus of the south-eastern part of the Paris Basin are particularly concerned with reform of CAP 92 and Agenda 2000, due to their overspecialization in wheat, barley and rapeseed. CAP 92 favoured income growth and gave administrative value to an area of modest potential yield («lands of stone »). But, CAP 92 is applied to a system of agricultural development in which increase of production at any price is the norm, and, without constant enlargement of the main agricultural uundertaking there is little room for manœuvre. This apparently prosperous agricultural region has become very dependant for its durability on external political decisions (CAP), while the flow of production has become more and more global. Consequently, it is hard to establish sustainable development in these fragile rural areas, like the Plateau de Langres, where agricultural activity is unavoidably part of the economic fabric. The resulting economy, linked to its dependance on the CAP, a condition of its present durability, poses a number of questions. This form of agriculture, a radically simplified countryside, is poorly matched to the logic of local sustainable development, in spite of rare examples to the contrary.

Die Ebenen im Südosten des Pariser Beckens sind wegen der Rotation, die sich nur noch auf Weizen, Gerste und Raps beschränkt, von der Reform der Gemeinsamen Agrarpolitik 1992 und von der Agenda 2000 besonders betroffen. Die gemeinsame Agrarpolitik hat sich auf die Einkommensverteilung günstig ausgewirkt, denn landwirtschaftlicher Boden mit mässigen Erträgen (karger steiniger Boden) hat dadurch einen administrativen Wert erhalten. Die Gemeinsame Agrarpolitik von 1992 fördert eine Entwicklung, in der eine übermässige Produktionssteigerung die Regel ist, in immer grösser werdenden Höfen, bei immer weniger werdenden Arbeitskräften. Diese scheinbar blühende Landwirtschaft ist von äusseren politischen Entscheidungen (Gemeinsame Agrarpolitik 1992) und von weltweiten Kursschwankungen in der Produktion abhängig geworden. Wie kann man eine nachhaltige Entwicklung in diesem landwirtschaftlichen Raum mit sehr grossen Anbauflächen in einer strukturschwachen ländlichen Gegend fördern, ohne dabei auf landwirtschaftliche Produktion zu verzichten ? Durch 
diese Art von Landwirtschaft, bei der viel zu viel investiert wurde, geht die Vielfalt in der Landschaft verloren. Das lässt sich schlecht vereinbaren mit Initiativen im ländlichen Bereich.

\section{INDEX}

Schlüsselwörter : Dauerhafte landwirtschaftliche Entwicklung, die auf dem Export basiert, Gemeinsame Agrarpolitik 92, grosse Anbauflächen, Landwirtschaft, landwirtschaftliche und ländliche Entwicklung, multifunktionnelle Landwirtschaft, nachhaltige Entwicklung, territorialisierte Anbaugebiete, umweltgerechte landwirtschaftliche Entwicklung Mots-clés : agriculture durable, agriculture raisonnée, Bassin parisien, contractualisation, développement agricole et rural, développement durable, grandes cultures, multifonctionnalité de l'agriculture, PAC 92, petites terres, système agro-exportateur, territorialisation des productions

Keywords : agro-exporting system, CAP 92, contractualisation, multi-functional agriculture, Paris Basin, sustainable agriculture, sustainable development, traceability

\section{AUTEUR}

\section{GENEVIÈVE PIERRE}

Université d'Angers - 35 rue de la Barre 49000 Angers. 